EP-29

\title{
Safety and effectiveness of non-use of operative site drainage after left lateral sectionectomy
}

\author{
Sunho OK, Hyungil SEO*, Youngmok PARK, Jaeri KIM, Myunghee YOON \\ Department of Surgery, Pusan National University Hospital, Busan, Korea
}

Introduction: Despite limited availability, prophylactic operative site drainage after elective hepatectomy has been used routinely. The objective of this study was to investigate the safety and effectiveness of nonuse of operative site drainage after left lateral sectionectomy.

Methods: Elective left lateral sectionectomy was performed 31 patients between Jan 2017 and June 2020. We retrospectively evaluate $\mathrm{d}$ data that were prospectively collected on left lateral sectionectomy. Patients were divided into drainage group $(\mathrm{n}=16)$ and non-use group $(\mathrm{n}=15)$.

Results: The patients included 22cases of hepatocellular carcinoma, 3cases of intrahepatic cholangiocarcinoma, 3cases of liver metastasis and 3cases of benign liver disease. Ten cases were performed by laparoscopic surgery. There was no difference between the two groups in clinicopathological factors and surgical outcomes such as incidence of surgical related complications. There was no case of intraperitoneal fluid collection requiring additional procedure after heaptectomy and early re-admission. But, in the period from surgery to discharge, the non-use group was significantly shorter than that of the drainage group (5.87 days vs. 8.44 days, $p=0.005$ ).

Conclusions: We concluded operative site drainage is unnecessary after elective left lateral sectionectomy. 